\title{
El Enfoque de la Conciencia Lingüística: La reflexión crítica en el aprendizaje y enseñanza de segundas lenguas
}

\section{The Language Awareness Approach: Critical Thinking in Second Lan- guage Acquisition}

\section{Ariadne de Villa}

Texas Lutheran University (TLU), EE.UU.

\section{Resumen}

Este artículo se centra en una actual investigación teórica con un marco descriptivo sobre la enseñanza de lenguas extranjeras y sobre cómo el Enfoque de la Conciencia Lingüística $(\mathrm{CL})$ es la clave para la enseñanza exitosa y el aprendizaje de un segundo idioma por su visión holística. La metodología de la CL proporciona al estudiante las herramientas y la confianza de interiorizar la segunda lengua para lograr una enseñanza permanente, durante el proceso de aprendizaje. A través del Enfoque de la $\mathrm{CL}$, los estudiantes se convierten en autodidactas altamente motivados, analizando debidamente la sintaxis de su lengua materna, así como la del segundo idioma. A medida que los estudiantes logran mayor habilidad en el entendimiento sobre el funcionamiento del lenguaje, son capaces de interiorizar una nueva lengua y hacerla suya. El artículo concluye proponiendo las metodologías de $L^{2}$ más aplicables en los tiempos contemporáneos.

Palabras clave: Lingüística aplicada; metodología de la adquisición de segundas lenguas; reflexión crítica; Enfoque de la Conciencia Lingüistica $(\mathrm{CL})$; educación bilingüe y plurilingüe; educación bicultural y multicultural.

\begin{abstract}
This paper reviews current theoretical research in second language methodology and on how the Language Awareness Approach, specifically, is key to successful second language teaching due to its holistic approach. LAA methodology gives students the tools and confidence to internalize the second language for the long term, by promoting the use of critical thinking. Through the Language Awareness Approach, students become highly motivated self-learners, expertly analyzing the syntax of their native language as well as that of the second language. As students become more skilled in understanding how language works, they are able to internalize the new language and make it their own. The paper closes by drawing conclusions of second language methodology appropriate for our contemporary times.

Keywords: Applied Linguistics; Second Language Education; Critical Thinking, Language Awareness Approach (LAA); bilingual and multilingual education; bicultural and multicultural education.
\end{abstract}

\section{METOdOLOGÍA DE LA ADQUISICIÓN DE LENGUAS}

En la actualidad, adquirir fluidez en más de un idioma, ya no es un lujo, sino se ha convertido en una necesidad. Es bien sabido que el inglés es la primera lengua más impartida en el mundo, siendo el mandarín, la segunda y el español, la tercera 
lengua más enseñada a nivel global. La necesidad de aprender un segundo idioma de forma permanente se ha vuelto vital. No obstante, el saber cómo aprenderlo es fundamental y por esta razón las diversas metodologías de la lingüística aplicada se han analizado continuamente: "Se ha estudiado en numerosas ocasiones la evolución de los paradigmas de enseñanza / aprendizaje de una segunda lengua a lo largo del siglo XX, hasta llegar al Enfoque Comunicativo o Funcional" (Marco y Zúñiga, 2014, p. 80). El Enfoque de la Conciencia Lingüística, cuyo objetivo es encontrar un equilibrio entre ambas metodologías, la estructuralista, la cual se enfoca en la gramática, y la comunicativa, la cual pone énfasis en la producción oral, es clave para una enseñanza y un aprendizaje práctico y permanente. El Enfoque de la Conciencia Lingüística además les proporciona al alumnado "la confianza en el dominio de patrones lingüísticos que sustenta la lengua" (Marco y Zúñiga, 2014, p. 81), al igual de proveerles una visión más amplia en el plano lingüístico y cultural.

El debate entre un modelo de enseñanza de la lengua en el que predomine la gramática y un modelo comunicativo que esté alejado de ella ha acompañada las últimas décadas de la enseñanza de lenguas extranjeras. No obstante tanto en las leyes educativas españolas como en las bibliografías que proponían un modelo de adquisición de la lengua sustentado en el modelo comunicativo, la gramática nunca quedaba olvidada en su planteamiento. Desde este nuevo punto de vista, no se trata de romper con todo el modelo anterior, sino, más bien, de llegar a él a través de otras perspectivas que lo completen (Marco y Zúñiga 2014, 80).

Las teorías sobre las lenguas atraviesan cambios constantes para servir mejor a las necesidades de los estudiantes de L2. A continuación abordaremos dos de los enfoques con más importancia por su uso actual en el contexto de la lingüística aplicada, el Enfoque Estructuralista y Comunicativo.

\section{EL ENFOQUE ESTRUCTURALISTA}

El Enfoque Estructuralista se definió por primera vez en el libro Curso de Lingüística General de Ferdinand de Saussure en 1916. Saussure fue el primer lingüista en aplicar una metodología científica en el estudio de la lengua. El autor indica que el lenguaje está compuesto de dos sistemas: la lengua y el habla (Iangue et parole). Para Saussure, la lengua es una estructura y un todo autónomo y por ende ha definido los conceptos de la misma desde un punto de vista sincrónico. La base de esta teoría es la sintaxis y la lengua como un sistema compuesto de subsistemas: el sistema fonológico, morfológico, el léxico y el sintáctico. 
Esta teoría excluye los aspectos socioculturales del idioma. Saussure sostiene que "solo la diferenciación entre relaciones sintagmáticas y asociativas puede proporcionar una clasificación que no está impuesta desde el exterior" al indicar lo siguiente:

Las agrupaciones en ambas clases son en su mayoría fijadas por el lenguaje; este conjunto de relaciones comunes constituye el lenguaje y dirige su funcionamiento. Según Saussure, "las solidaridades sintagmáticas y asociativas son lo que limita la arbitrariedad y otorga motivación" (Saussure, 1967, p. 132).

El autor pone así de relieve la estructura gramatical como el objetivo principal de su metodología. Por lo tanto, esta misma ha conducido a una aproximación basada en el texto desde que la gramática y su estructura son sus puntos principales. Europa adoptó en primera instancia este método de enseñanza de segundas lenguas, el cual se sigue utilizando en la actualidad. Siguiendo los principios básicos del estructuralismo, se creó una metodología de enseñanza de $L^{2}$ que revolucionó dicho campo y anuló casi por completo el método de gramática-traducción, vigente hasta los años 40 del siglo XX. Aún quedan huellas importantes del enfoque estructuralista, las cuales se pueden apreciar en la actualidad, tales como la mayoría de los ejercicios que forman parte de los libros de texto de $L^{2}$, sin importar el enfoque metodológico en el cual esté basado el libro.

\section{EL ENFOQUE COMUNICATIVO}

En contraste con el Enfoque Estructuralista, el Enfoque Comunicativo o Funcional emergió en 1967 con la influencia del lingüista Noam Chomsky en Estados Unidos. Pilar Melero Abadía lo resume de la siguiente manera en el prólogo de "El enfoque comunicativo" en las Antologías de textos de didáctica del español:

A mediados de los años 60 se empezó a rechazar la teoría lingüística estructural
y el aprendizaje basado en la concepción conductista. Se vislumbraba que el
objetivo del proceso de enseñanza-aprendizaje tenía que ir encaminado hacia el
desarrollo de la competencia comunicativa y no solo de la competencia lingüística;
es decir, que los alumnos fueran capaces de comunicarse de forma adecuada en
la lengua extranjera y que la enseñanza de la gramática estuviera subordinada al
uso de la lengua con fines comunicativos (Abadía, Introducción, p. 1).

Esta metodología surgió del área de la sociolingüística, la cual subrayó que la necesidad de la comunicación en un contexto específico es una oportunidad para practicar y aprender el idioma en proceso de aprendizaje: Se define por su conocido lema: "La comunicación es la clave". 
El Enfoque Comunicativo se originó como reacción frente a la Teoría Estructuralista que posee un "enfoque basado en el texto más que una focalización sobre el proceso implicado" (Richards y Rodgers, 1995, p. 41). Este movimiento, tal y como lo describió Richards "mira las implicaciones más amplias de considerar a hablantes y oyentes como seres sociales, que funcionan en un contexto que es a la vez personal, conceptual e interpersonal" (Richards y Rodgers, 1995, Introducción). Por ende, el Enfoque Comunicativo es conocido por su objetivo comunicativo y no por la estructura gramatical de la propia lengua. El Enfoque Comunicativo, posterior a utilizarse en el continente americano, con especial énfasis en los Estados Unidos, desarrolló un papel importante en el Consejo de Europa en el año 1971.

La competencia comunicativa es uno de los conceptos clave en la enseñanza de idiomas en la actualidad. Hymes (1971) puso en relieve la importancia de la competencia comunicativa en contraste con la competencia lingüística de Saussure. Asimismo, cuestionó el rol del alumno y sus necesidades, lo cual originó el taskbased approach o método por tareas. Fue así como el enfoque dentro del aula evolucionó al convertirse el mismo en student-centered, en el cual el estudiantado y no el profesor, es centro de atención en el aula: "De ese modo, los aprendientes pueden hacer aflorar en el discurso en el aula sus intuiciones sobre la organización y funcionamiento del sistema lingüístico, sobre sus propias necesidades de aprendizaje y sobre la forma de satisfacerlas." La evolución del método por tareas ha llevado a la primacía en las últimas décadas el cual propone distintas actividades ya sea individuales o en grupo, las cuales promuevan el uso de la lengua en registros oral y escrito. Este enfoque tendría como forma de trabajo privilegiado la elaboración de proyectos. Su objetivo es fomentar el aprendizaje mediante el uso de la lengua emulando contextos reales en el aula y no únicamente mediante la manipulación de unidades de sus diversos niveles de descripción. Es así como los procesos de aprendizaje incluirán necesariamente procesos de comunicación y el conocimiento cultural de la lengua.

Al proponer contextos de uso para las estructuras que se van utilizando, evita que se fijen errores lingüísticos, se trabaja deduciendo la norma de manera flexible a partir de la multiplicidad de ocasiones en la cual se usa la misma (Marco y Zúñiga, 2014, p. 80). De una forma paralela, la autora Josette Jolibert, sostiene la importancia de la presencia de una mayor flexibilidad por parte del docente hacia el aprendiente en distintos contextos: "la reconciliación gozosa de los estudiantes y de los docentes con su lengua; la construcción de competencias tanto comunicativas como lingüísticas diversificadas; la capacidad de evaluar una situación y de tomar decisiones pertinentes, de resolver problemas por la negociación más que por la 
agresión o el silencio; la voluntad de defender y usar su poder recién conquistado, y, como consecuencia, la construcción de competencias didácticas y pedagógicas pertinentes y en perpetuo proceso de renovación" (Jolibert, 2000, p. 13).

\title{
4. EL ENFOQUE DE LA CONCIENCIA LINGÜÍSTICA (CL)
}

El tercer enfoque que abordaremos es el de la Conciencia Lingüística (CL), el cual emergió en los años setenta. Según la ALA (Association for Language Awareness), consiste en "el conocimiento explícito acerca de la lengua y la percepción y sensibilidad conscientes al aprender la misma, al enseñarla y al usarla." Según la información que nos proporciona el Instituto Cervantes, el Enfoque de la Conciencia Lingüística está relacionada con el particular propósito de aprender. Este enfoque implica el acceso al conocimiento que el estudiante posee sobre la propia noción de la lengua, y "así se distancia de los planteamientos conductistas en la enseñanza de lenguas" (43). El Enfoque Comunicativo no respondía a las necesidades docentes ya que el objetivo comunicativo era válido, sin embargo, los estudiantes no contaban con las herramientas necesarias para realizar esta comunicación. Asimismo, se encontró cierta problemática con la omisión de la enseñanza formal de la lengua. Cots, Armengol, Arnó, Irún y Llurda describen de la siguiente manera el surgimiento del Enfoque de la Conciencia Lingüística en la enseñanza de lenguas:

\begin{abstract}
Los métodos comunicativos que han predominado durante las dos últimas décadas, en que el énfasis se centraba casi de manera exclusiva en la transmisión de significado, se están revaluando con respecto al papel que ha de tener la atención a los aspectos formales de la lengua. De hecho el componente metalingüístico que había estado presente en los métodos tradicionales de enseñanza de lenguas quedó relegado en la aplicación del enfoque comunicativo. En este sentido, se intentaba promover la capacidad comunicativa del aprendiente a través de actividades centradas en el significado, lo que implicaba que se dejara bastante de lado la atención a los aspectos formales (Cots, Armengol, Arnó, Irún y Llurda, 2007, p. 19).
\end{abstract}

La lingüística aplicada había ido reflexionando sobre la importancia y la necesidad de englobar dentro de los Enfoques Comunicativos, la conciencia lingüística o la reflexión sobre la lengua y la comunicación (Marco y Zúñiga, 2014, p. 81). Esta perspectiva, en gran parte fue definida gracias a los trabajos de Stephen Krashen en el año 1982, los cuales presentaron esta visión por primera vez. Puesto que el componente metalingüístico en la enseñanza de lenguas es esencial, la propuesta de la utilización del Enfoque de la Conciencia Lingüística puede tener validez. Este enfoque ofrece un equilibrio entre el Enfoque Estructuralista y el Enfoque Comuni- 
cativo, donde la reflexión crítica y la interiorización de la lengua sirven como recurso para ayudar a los estudiantes a aprender y comprender el idioma a corto y largo plazo. Asimismo, el Enfoque de la Conciencia Lingüística le otorga al estudiantado las herramientas necesarias para solucionar cognitivamente el problema comunicativo en distintos contextos. Este empoderamiento por parte del estudiante ha resultado en una alta motivación propia para resolver el problema comunicativo mediante el análisis crítico de la lengua.

La sensibilización de la lengua (SL) tomado de la expresión inglesa consciousness raising, es un término secundario para referirse al Enfoque de la Conciencia Lingüística. Se presenta como un intento deliberado de llamar la atención explícita del estudiante hacia rasgos en la segunda lengua, particularmente los aspectos gramaticales. Esta idea pudiese asemejarse a la corriente estructuralista de Saussure, ampliamente utilizada en Europa. No obstante, Rutherford insiste en que la misma difiere de la siguiente manera: "la sensibilización de la lengua (SL) es un medio de alcanzar la competencia gramatical... mientras que "enseñar gramática" representa típicamente un intento de infundir la competencia lingüística de manera directa" (Rutherford 1987, p. 24). Asimismo, la sensibilización de la lengua posee un enfoque explícito acerca de la gramática como necesaria pero no suficiente para desarrollar la competencia lingüística, mientras que la enseñanza tradicional de la gramática la considera como necesaria y suficiente. Además reconoce el papel activo del estudiante en la construcción de la gramática; la enseñanza tradicional de la gramática considera al estudiante como una tabla rasa, una pizarra en blanco. Finalmente, la enseñanza tradicional de la gramática se centra principalmente en la sintaxis, mientras que el Enfoque de la Conciencia Lingüística no solo se centra en la sintaxis, sino también en su relación con la semántica.

El Enfoque de la Conciencia Lingüística considera la gramática como un componente explícito para interiorizar la lengua, pero no como único recurso. Es aquí donde la reflexión crítica posee un rol importante en el proceso del aprendizaje de un segundo idioma. Esta metodología alienta al alumno a utilizar la reflexión crítica en la vida cotidiana, sin estar limitado al contexto del aula. El objetivo de este enfoque es que el profesorado dote a los estudiantes con las estrategias necesarias para que se auto motiven y puedan también analizar el idioma en una situación comunicativa mediante el uso de la reflexión crítica de la lengua. Bolitho y otros afirman que el elemento clave del Enfoque de la Conciencia Lingüística es que los estudiantes "descubran el idioma por sí mismos" (2003, p. 251). Hawkins menciona que el Enfoque de la Conciencia Lingüística anima a los estudiantes "a realizarse preguntas sobre la lengua" y como resultado "recopilan su propia infor- 
mación del mundo exterior y asimismo dentro del aula" (Hawkins 1984, p. 4-5). Como resultado, los estudiantes aprenden a reflexionar por sí mismos y a la vez son capaces de resolver una situación comunicativa en distintos contextos (formales e informales), partiendo de su propio conocimiento, su lengua y el análisis crítico.

A medida que los aprendices de una segunda lengua incrementan su competencia lingüística cuando utilizan el análisis de la lengua para resolver una situación comunicativa, el alumnado se auto motiva y continúa practicando el lenguaje fuera del aula, en distintos contextos: "el efecto de todo esto en la autoestima de los alumnos, a medida que se convierten en ser sus propios expertos más que apoyarse en el conocimiento recibido en el aula" (Bolitho y otros 2003, p. 255). Mediante este método, los estudiantes incrementan su autoconfianza por las siguientes dos razones: La primera es que los estudiantes son capaces de la producción de la lengua en un contexto específico. La segunda razón es que interiorizan el idioma como resultado de este proceso de análisis, lo cual tiene como resultado el aprendizaje de la lengua a largo plazo y no a corto como si fuera una simple actividad de memorización. La tercera es que no dependen del profesorado para resolver una situación comunicativa y por ende esto implica un incremento de autoconfianza en el alumnado. Como consecuencia, la auto motivación les anima a los estudiantes a perseverar con el proceso de aprendizaje de la lengua.

El papel de la afectividad en el Enfoque de la Conciencia Lingüística causó el impacto en el estudiantado de convertirse en motivados analistas de la lengua para solucionar una situación comunicativa. Sin embargo, con el fin de realizar esta acción, las investigaciones muestran que los instructores en el aula poseen indudablemente un papel fundamental en la motivación del estudiante (Bolitho y otros, 2003, p. 251). Este papel del formador es sin dudas la clave para crear un ambiente positivo en el salón de clases mientras se les facilitan las herramientas que apoyen el desarrollo de las habilidades de reflexión crítica necesaria para analizar el lenguaje en una situación comunicativa. El Sprachgefüh/ traducido como el sentimiento intuitivo de la utilización de lenguaje o bien, la intuición lingüística, es una señal importante de motivación y éxito de los estudiantes a la hora de aprender una segunda lengua. Según Tomlinson, el Enfoque de la Conciencia Lingüística "abre las puertas a estas dimensiones afectivas bajo caminos que pueden hacer la diferencia entre los estudiantes" (1994, p. 256). Esta metodología en combinación con una instrucción efectiva, refuerzo positivo y una implicación emotiva, aumenta los caminos neuronales en múltiples áreas del cerebro, logrando la representación multidimensional necesaria para un proceso profundo del lenguaje (1994, p. 256). 
Finalmente, el Enfoque de la Conciencia Lingüística va paralelo a la definición de la lengua y su propósito. Según el diccionario Merriam-Webster, la definición de la lengua es la siguiente: "las palabras, su pronunciación, y los métodos de combinarlos usados y comprendidos por una comunidad" (Merriam-Webster, 2017). Por ende, la lengua surge de forma natural y que está integrado en nosotros mismos (Kumaravadivelu, 2006, 156). Steven Pinker afirma que la gente no es consciente de esta habilidad natural de la producción lingüística y lo afirma de la siguiente manera: "A medida que lees estas palabras, estás formado parte de una de las maravillas del mundo natural, puesto que tú y yo pertenecemos a una especie dotada con esta notable habilidad... Esta habilidad es la lengua... Surge tan naturalmente que nos permitimos olvidar el milagro que supone" (Pinker, 1994, 15).

Puesto que la lengua es un proceso natural en los seres humanos, el Enfoque de la Conciencia Lingüística utiliza esta habilidad y la combina con el lenguaje, con una actitud positiva y con la metacognición para una producción de la L². Van Lier cita a Humboldt: "El lenguaje no puede ser realmente enseñando. Tan solo puede ser despertado en el alma," haciendo referencia a este proceso natural interno del hombre. (1995, p. 21). Chomsky realiza una clara distinción entre I-language o lenguaje interno y E-language o lenguaje externo. Según Van Lier, "esta distinción refleja la dicotomía entre competencia y realización" (1995, p. 20). I-language corresponde al lenguaje estandarizado, innato en el ser humano, el órgano lingüístico, según Chomsky. Este es el lenguaje que según Chomsky debe considerarse como primordial puesto que va paralelo a la naturaleza del ser humano. El Enfoque de la Conciencia Lingüística, por ende, considera la naturaleza del ser humano en la facilidad que indica aprender una lengua y mediante el pensamiento crítico de la propia lengua se le proveen a los estudiantes las herramientas para resolver una situación comunicativa en un contexto sociocultural específico.

En resumen, el método de Conciencia Lingüística provee una visión más holística entre la teoría Estructuralista de Saussure la cual incorpora la gramática al aula y el Enfoque Comunicativo de Chomsky que tiene la comunicación como objetivo principal. El Enfoque de la Conciencia Lingüística, por ende utiliza el análisis de estructuras gramaticales junto con la reflexión crítica con el fin de lograr una comunicación eficaz, y por lo tanto sirve como un recurso natural para los seres humanos.

Según varias investigaciones lingüísticas, se ha comprobado en diversas ocasiones el interés que ha despertado el enfoque de la conciencia lingüística puesto que se ha comprobado una existente "correlación positiva y significativa entre los logros en el aprendizaje de la lectoescritura y la conciencia léxica y fonológica" (Manri- 
que y Signorini, 1997, p. 1). Ehri indica que "es evidente que el lenguaje escrito proporciona esquemas para conceptualizar y analizar la estructura del habla. La conciencia lingüística interactúa con el proceso de aprendizaje de la lectoescritura porque es tanto una consecuencia como una causa que facilita el progreso de este progreso (1975, p. 212). En el trabajo de Manrique y Gramigna (1987) se dio a conocer cómo la reflexión de la lengua mediante diversas ejercitaciones orales de análisis y síntesis de palabras y oraciones a niños, tuvo como resultado un incremento significativo en las habilidades analíticas, derivado sin duda, de los ejercicios de segmentación léxica y fonológica" (1987, p.3). Según estas investigaciones puede deducirse que en Enfoque de la Conciencia Lingüística es una herramienta útil e importante en tanto en el aprendizaje como en la adquisición de segundas lenguas en niños y adultos.

En conclusión, con la creciente demanda de aprender una segunda lengua a nivel global, las metodologías de la enseñanza de lenguas están sufriendo cambios constantes para atender las necesidades de los aprendientes. Al tener presente la importancia de resolver una situación comunicativa en distintos contextos, sin ignorar los aspectos socioculturales del entorno, así como la capacidad de encontrar intuitivamente soluciones a la estructura del lenguaje de manera correcta con el fin de dotarla del significado adecuado, el Enfoque de Conciencia Lingüística dota de un efecto holístico en el aprendizaje y la enseñanza de lenguas. Si partimos del concepto del Enfoque de la Conciencia Lingüística, el cual sostiene que el aprendizaje de una lengua es una parte innata del ser humano, mediante la reflexión crítica, se vuelve una atractiva y eficaz metodología para la enseñanza de segundas lenguas.

El resultado de la utilización de este enfoque es la obtención de una segunda lengua con fluidez, una motivación intrínseca y las estrategias que el estudiantado obtiene para resolver la comunicación mediante el uso del razonamiento crítico y el análisis de su propia lengua. Algunos autores han enfatizado las aportaciones del Enfoque de la Conciencia Lingüística según Van Lier (1996): En las cuestiones afectivas, la CL despierta la sensibilidad y curiosidad por aprender. En cuanto la dimensión cognitiva, la relación entre pensamiento y lenguaje "como desarrollo de la capacidad de análisis de los aprendientes: dicha capacidad se estimula en el estudio de la lengua y es fácilmente extrapolable a otras áreas de conocimiento" (1996). En cuanto a la producción oral y escrita de la lengua, el conocimiento que se haya adquirido mediante el enfoque $\mathrm{CL}$, conduce a una mejora en la utilización de la lengua reflejado tanto en el registro oral, como en el escrito. Finalmente, el Enfoque de la Conciencia Lingüística, "redunda en una mayor tolerancia y respeto por otras lenguas y otras variedades, en una mejora de las relaciones entre grupos 
étnicos y en le incremente del plurilingüismo y la pluriculturalidad" (Fairclough, 1995). Se ha podido observar que los aprendientes de segundas lenguas que utilizan el enfoque de la CL gozan de un mayor poder de adaptación a nivel cultural por el uso de la reflexión crítica. Por consiguiente, el Enfoque de la Conciencia Lingüística es una propuesta de carácter metodológico, cuyo objetivo es encontrar un equilibrio entre ambos métodos: el Estructuralista y el Comunicativo o Funcional. El mismo le proporciona al alumnado, las herramientas necesarias para resolver una situación comunicativa mediante la reflexión crítica en distintos contextos. Mediante este enfoque, el estudiantado se motiva y se vuelve autosuficiente en el proceso de aprendizaje y adquiere un conocimiento global de otras lenguas y sus variedades, lo cual le permite tener una visión más profunda a nivel lingüístico y cultural, idóneo para nuestro mundo actual.

\section{BIBLIOGRAFÍA}

Abadía, Pilar M. (2018). Enfoque comunicativo. Antologías didácticas. Centro Virtual Cervantes. Disponible en: https://cvc.cervantes.es/ensenanza/biblioteca ele/ antologia didactica/enfoque comunicativo/introduccion01.htm

Bolitho, R., Carter, R., Hughes, R., Ivanic, R., Mashura, H., and Tomlinson, B. (2003). Ten Questions about Language Awareness, Oxford University Press.

Cots, J., Armengol, L., Arnó, E., Irún, M., \& Llurda, E. (2007). La conciencia lingüística en la enseñanza de lenguas. Barcelona: Graó.

Ehri, L. (1975). Word Consciousness in Readers and Prereaders. Journal of Educational Psychology.

Fairclough, N. (1995). Critical Discourse Analysis. Londres: Longman.

Hawkins, Eric (1984). Awareness of Language: An Introduction. Cambridge, New York, Cambridge University Press.

Hymes, Dell (1971). Thirty Years of Linguistic Evolution: The concept of communicative competence revisited. University of Virginia, Charlottesville, U.S.A.

Instituto Cervantes. (2017). Conciencia Lingüística, Centro Virtual Cervantes. Diccionario de términos ELE. Consultado en: http://cvc.cervantes.es/ensenanza/biblioteca ele/ diccionario/conciencialinguistica.htm

Jolibert, Josette. (2000). “¿Mejorar o Transformar "de veras" la formación docente? Aspectos críticos y ejes clave." Lectura y vida.

Krashen, S. Principles and Practice in Second Language Acquisition (1982). Oxford: Pergamon Press Inc., University of Southern California. 
Kumaravadivelu, B. (2006). Understanding Language Teaching. From Method to Postmethod. San Jose State University, Lawrence Erlbaum Associates, Publishes. Mahwah: New Jersey.

Manrique y Gramigna. (1987). Función de la conciencia lingüística en el aprendizaje de la lectoescritura.

Manrique A. y Signiorini A. (1997). Del habla a la escritura. La conciencia lingüística como una forma de transición natural. Lectura y vida.

Marco, M. y Zúñiga, E. (2014). La conciencia lingüística en la enseñanza del español como lengua extranjera, Publicaciones Didácticas.

Merriam-Webster Dictionary of Language. (2016). Definition of Language. http://www. merriam-webster.com/dictionary/language

Pinker, S. (1994). The Language Instinct. New York, NY: Harper Perennial Modern Classics.

Richards, J.C. y Rodgers, T. (1995) Enfoques y métodos en la enseñanza de idiomas. Madrid: Cambridge University Press.

Rutherford, William. (1987). The meaning of grammatical consciousness-raising. Wiley Online Library. Consultado en: https://onlinelibrary.wiley.com/doi/abs/10.1111/j.1467971X.1987.tb00201.x

Saussure, F. de (1967). Curso de Lingüística General. Buenos Aires: Losada.

Tomlinson, B. (1994). Pragmatic awareness activities. Language Awareness. 3/3.

Van Lier, L. (1998). «La relación entre concienciación, interacción y aprendizaje de lenguas». En Cots y Nussbaum, 2000.

Van Lier, L. (1995) «Lingüística Educativa. Una introducción para enseñantes de lenguas». Signos. Teoría y práctica de la educación. 
